\title{
Reversible Capacity of Conductive Carbon Additives at Low Potentials: Caveats for Testing Alternative Anode Materials for Li-Ion Batteries
}

\author{
Kimberly A. See, ${ }^{\mathrm{a}, \mathrm{b}, \mathrm{c}, *, \mathrm{z}}$ Margaret A. Lumley, ${ }^{\mathrm{b}, \mathrm{c}}$ Galen D. Stucky, ${ }^{\mathrm{b}, \mathrm{c}, \mathrm{d}, \mathrm{e}}$ Clare P. Grey, ${ }^{\mathrm{f}, *}$ \\ and Ram Seshadri ${ }^{b, c, d, e, z}$ \\ ${ }^{a}$ Department of Chemistry, University of Illinois at Urbana-Champaign, Urbana, Illinois 61801, USA \\ ${ }^{b}$ Mitsubishi Chemical - Center for Advanced Materials, University of California, Santa Barbara, \\ California 93106, USA \\ ${ }^{c}$ Department of Chemistry and Biochemistry, University of California, Santa Barbara, California 93106, USA \\ ${ }^{d}$ Materials Research Laboratory, University of California, Santa Barbara, California 93106, USA \\ ${ }^{e}$ Materials Department, University of California, Santa Barbara, California 93106, USA \\ ${ }^{f}$ Department of Chemistry, University of Cambridge, Lensfield Road, Cambridge CB2 1EW, United Kingdom
}

\begin{abstract}
The electrochemical performance of alternative anode materials for Li-ion batteries is often measured using composite electrodes consisting of active material and conductive carbon additives. Cycling of these composite electrodes at low voltages demonstrates charge storage at the operating potentials of viable anodes, however, the conductive carbon additive is also able to store charge in the low potential regime. The contribution of the conductive carbon additives to the observed capacity is often neglected when interpreting the electrochemical performance of electrodes. To provide a reference for the contribution of the carbons to the observed capacity, we report the charge storage behavior of two common conductive carbon additives Super P and Ketjenblack as a function of voltage, rate, and electrolyte composition. Both carbons exhibit substantial capacities after 100 cycles, up to $150 \mathrm{mAh} \mathrm{g}^{-1}$, when cycled to $10 \mathrm{mV}$. The capacity is dependent on the discharge cutoff voltage and cycling rate with some dependence on electrolyte composition. The first few cycles are dominated by the formation of the SEI followed by a fade to a steady, reversible capacity thereafter. Neglecting the capacity of the carbon additive can lead to significant errors in the estimation of charge storage capabilities of the active material.

(C) The Author(s) 2016. Published by ECS. This is an open access article distributed under the terms of the Creative Commons Attribution Non-Commercial No Derivatives 4.0 License (CC BY-NC-ND, http://creativecommons.org/licenses/by-nc-nd/4.0/), which permits non-commercial reuse, distribution, and reproduction in any medium, provided the original work is not changed in any way and is properly cited. For permission for commercial reuse, please email: oa@electrochem.org. [DOI: 10.1149/2.0971702jes] All rights reserved.

(cc) BY-NC-ND
\end{abstract}

Manuscript submitted November 2, 2016; revised manuscript received December 15, 2016. Published December 29, 2016.

The investigation of alternative anode materials for Li batteries is driven by the need for sustainable materials exhibiting high, reversible capacity at low voltages that outperform the current ubiquitous anode chemistry provided by $\mathrm{Li}$ intercalated graphite. Although the Li-ion battery is currently capacity limited by the cathode, next-generation cathodes such as elemental $S_{8}$, for example, have high theoretical capacities, up to five times higher than the conventional lithiated graphite anode. Due to this capacity mismatch, Li-S cells rely on the use of unsafe Li metal anodes that add significant challenges to the already complicated cathode chemistry to achieve high energy densities.

Systems evaluated as high capacity anodes include elemental electrode materials such as $\mathrm{Si}_{1,2}, 2$ and conversion materials including transition metal oxides, ${ }^{3-5}$ fluorides,${ }^{6,7}$ hydrides, ${ }^{8}$ and sulfides. ${ }^{9,10}$ Conversion materials are of interest because the full oxidation state of the transition metal can be utilized, enabling high theoretical capacities. The electrochemical activity of alternative anode materials is often evaluated in composite electrodes at low potentials, close to that of $\mathrm{Li} / \mathrm{Li}^{+}$, to demonstrate charge storage at viable anode potentials. The composite electrodes contain varying percentages of conductive carbon and polymeric binder in addition to the active material, as shown schematically in Figure 1, in order to enhance the conductivity and mechanical stability of the electrode. ${ }^{11}$ The capacity of the electrode is usually dominated by the charge storage behavior of the active material. However, the charge storage abilities of the slurry could arise from a combination of the capacity of all three components (Figure $1)$.

In some cases, the contribution of the conductive carbons to the observed capacity of the electrode is significant and cannot be neglected. Such contributions have been suggested previously, ${ }^{12-14}$ but in practice they are not always quantified and analyzed in any great detail as a function of, for example, rate, electrolyte composition, etc.

*Electrochemical Society Member.

zE-mail: ksee@illinois.edu; seshadri@mrl.ucsb.edu
A previous study on a low surface area carbon black $\left(80 \mathrm{~m}^{2} \mathrm{~g}^{-1}\right)$ reports a reversible capacity of $180 \mathrm{mAh} \mathrm{g}^{-1}$ after 10 cycles in a $\mathrm{LiBF}_{4}$ electrolyte further confirming the utility of a systetmatic study of the reversible capacity associated with the carbon additives. ${ }^{14}$ Situations in which carbons could contribute significant capacities relative to the active material include electrode slurries that contain a large
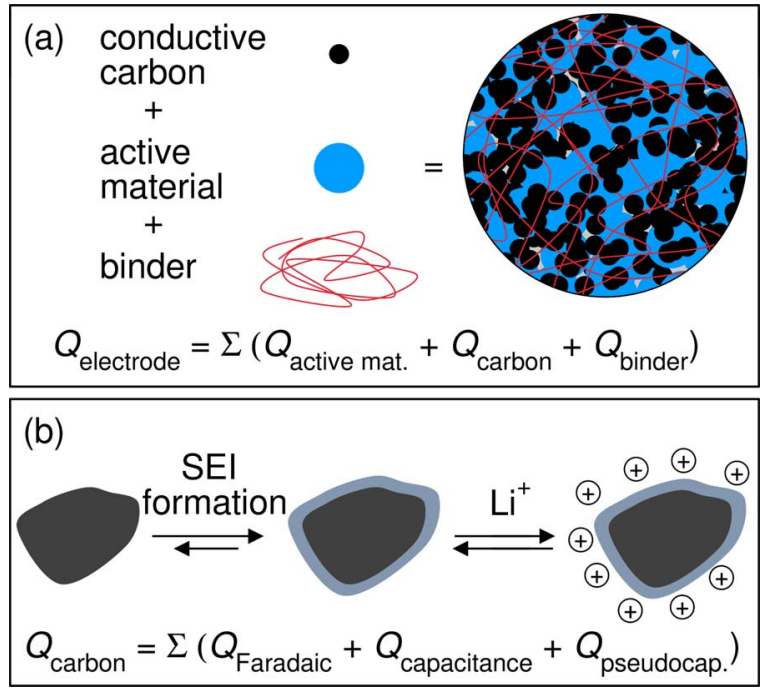

Figure 1. (a) Alternative anode materials are commonly evaluated in slurry electrodes consisting of the active material, conductive carbon additives, and binder. The capacity of slurry electrodes can arise as a result of the charge storage capabilities of all three components. (b) Carbon additives can store charge at low voltages via faradaic mechanisms including solid-electrolyte interface (SEI) formation and $\mathrm{Li}^{+}$intercalation (not shown) in addition to capacitive and pseudocapacitive charge storage. 
percentage of carbon, (which can comprise up to $50 \%$ when highly insulating materials such as some oxides are examined) and electrodes that are cycled at low potentials, where carbon materials are able to reversibly store charge. Understanding the magnitude of this contribution is necessary to evaluate the activity of the active material itself accurately.

Carbons can store charge at low potentials through a variety of mechanisms including faradaic, capacitive, and pseudocapacitive charge storage and the distinctions between the different mechanisms are often blurred and difficult to determine (Figure 1b). The activity of carbons is well established as carbonaceous materials are widely used in electrochemical energy storage devices as the active materials themselves. The mechanism of charge storage, quantity of irreversible capacity, and voltage hysteresis depend on the degree of graphitization in the carbon. ${ }^{15}$ Ordered carbons such as the conventional Li-ion intercalation graphite anode for example, can store around $350 \mathrm{mAh} \mathrm{g}^{-1}$ via faradaic intercalation chemistry, ${ }^{16}$ while amorphous, high surface area carbons are often used as the active materials in supercapacitors ${ }^{17}$ and can achieve capacities around $250 \mathrm{~F} \mathrm{~g}^{-1} .{ }^{18}$ The conductive carbon additives used in electrode slurries are largely amorphous and the surface areas are often 10-20 times smaller than those used in supercapacitor electrodes. Amorphous carbons can exhibit graphitic character depending on the pyrolysis temperature, allowing for the possibility of intercalative storage. Faradaic intercalation is normally signaled by a plateau in the galvanostatic discharge and charge profiles. In amorphous carbons, intercalation can manifest as a sloping profile due to the wide distribution of chemical environments. ${ }^{19}$ As the carbons become less ordered, the ability of the materials to intercalate $\mathrm{Li}^{+}$becomes more dependent on electrolyte composition. ${ }^{20}$ Additional contributions due to capacitive storage, pseudo-capacitive storage, and quasi-reversible electrolyte decomposition (reversible SEI formation) have also been observed. ${ }^{21-23}$ Double-layer capacitance tends to scale with surface area and the quantity of $\mathrm{H}$ in the material ${ }^{22}$ suggesting that this storage may be site specific and slightly pseudocapacitive. Amorphous carbons have even been evaluated as possible alternatives to the ordered graphite anode with the advantage of better rate capabilites compared to ordered carbons. ${ }^{24}$ At high voltages $(>4 \mathrm{~V})$, anion intercalation has been observed in amorphous carbons ${ }^{25}$ in addition to electrolyte decomposition. ${ }^{26}$

The variety of mechanisms by which amorphous carbons can contribute to the observed capacity at low potentials necessitates a systematic study that quantifies their capacity under cycling conditions used to evaluate alternative anode materials, i.e. in carbonate electrolytes at low voltages with a variety of scan rates. Understanding the contribution of the carbon additives will enable a more accurate interpretation of the electrochemical behavior of alternative anodes. Here, we describe the activity of two common additives: Super P (TIMCAL) and Ketjenblack (AkzoNobel). The particle sizes of Super P and Ketjenblack are similar, around $40 \mathrm{~nm}^{27}$ and $30 \mathrm{~nm},{ }^{28}$ respectively. Despite their similar sizes, Ketjenblack has a much higher specific surface area of $1447 \mathrm{~m}^{2} \mathrm{~g}^{-128}$ compared to that of Super P at $62 \mathrm{~m}^{2} \mathrm{~g}^{-1}$, according to the TIMCAL technical data sheet. The higher surface area of Ketjenblack is due to the hollow morphology of the particles resulting in additional internal surface area. ${ }^{28}$ Although the physical surface areas of the two carbons are very different, it is important to note that the entire surface area is not necessarily electrochemically accessible.

We find here that Super P and Ketjenblack exhibit substantial reversible capacities, between $100 \mathrm{mAh} \mathrm{g}^{-1}$ to $175 \mathrm{mAh} \mathrm{g}^{-1}$, when cycled in carbonate electrolytes at low potentials, defined as below $1 \mathrm{~V}$ vs. $\mathrm{Li} / \mathrm{Li}^{+}$for this study. The stable, reversible capacity is, of course, preceded by an exceedingly large first discharge capacity due to decomposition of the electrolyte to form the solid electrolyte interphase (SEI). ${ }^{29-31}$ The composition of the SEI on Super P has recently been evaluated using solid-state NMR. ${ }^{23}$ The high, reversible capacity of subsequent cycles, however, has not yet been quantified. This reversible capacity is important to consider when cycling alternative anode materials that function at low voltages in composite electrodes. Although the contribution of the carbon material to the overall electrode performance depends on a variety of factors that include elec- trode composition, electrode preparation method, and the nature of the active material, it is our goal to provide an estimate of the highest possible reversible capacity of conductive carbon additives to be used as a reference when evaluating the performance of alternative anode materials.

\section{Experimental}

Prior to cell testing, Super $\mathrm{P}$ and Ketjenblack were ground with a mortar and pestle and dried in a vacuum oven at $120^{\circ} \mathrm{C}$ for $24 \mathrm{hrs}$. The electrolyte solvents ethylene carbonate (EC), ethyl methyl carbonate (EMC), dimethyl carbonate (DMC), and diethyl carbonate (DEC), were purchased anhydrous and used as received. Battery grade lithium hexafluorophosphate $\left(\mathrm{LiPF}_{6}\right)$ was used as received. All electrolyte solutions were prepared in an Ar glove box with $1 \mathrm{M} \mathrm{LiPF}_{6}$ and were stirred for at least $24 \mathrm{hrs}$ before use.

Stainless steel, 1/2" Swagelok cells with Kapton liners were used to evaluate the electrochemical activity of the carbons. The cells were assembled in an Ar glove box using $2 \mathrm{mg}-4 \mathrm{mg}$ of carbon added directly to the stainless steel plunger. The carbons were separated from the $10 \mathrm{~mm} \mathrm{Li}$ foil anode with two 1/2" Whatman glass fiber (GFD) separators. The GFD separators were dried at $70^{\circ} \mathrm{C}$ under vacuum overnight prior to loading into the glove box. Ten drops of electrolyte were added with a glass pipette to the top of the separators prior to placing the Li metal foil anode and stainless steel current collector.

Electrochemical measurements were performed on a Bio-Logic Variable Multichannel Potentiostat. Galvanostatic cycling was performed with a charging cutoff voltage of $2.0 \mathrm{~V}\left(\mathrm{vs} . \mathrm{Li}^{\prime} / \mathrm{Li}^{+}\right)$and varying discharge cutoff voltage between $0 \mathrm{~V}\left(\mathrm{vs} . \mathrm{Li} / \mathrm{Li}^{+}\right)$to $0.5 \mathrm{~V}\left(\mathrm{vs} . \mathrm{Li} / \mathrm{Li}^{+}\right.$). All subsequent references to voltage are vs. $\mathrm{Li} / \mathrm{Li}^{+}$. The cycling rate was varied between $3.72 \mathrm{~A} \mathrm{~g}^{-1}, 0.744 \mathrm{~A} \mathrm{~g}^{-1}, 0.372 \mathrm{~A} \mathrm{~g}^{-1}$, and $0.0372 \mathrm{~A} \mathrm{~g}^{-1}$ corresponding to $10 C, 2 C, C$, and $C / 10$, respectively, assuming $1 \mathrm{e}^{-}$per $6 \mathrm{C}$. Electrochemical impedance spectroscopy (EIS) was performed before and after cell cycling with a sinusoidal amplitude of $100 \mathrm{mV}$ from $1000 \mathrm{kHz}$ to $10 \mathrm{mHz}$.

\section{Results and Discussion}

The goal of this study is to provide an estimate of the reversible capacity expected for two common carbon additives when cycled at low potentials in a variety of electrolytes at several rates. The carbons are evaluated as loose powder electrodes compressed in a Swagelok cell, allowing us to forgo the use of binder and evaluate the carbons themselves. Firstly, we will discuss the effect of the discharge cutoff voltage on the reversible capacity of Ketjenblack. Figure 2 shows the behavior of Ketjenblack electrodes discharged to $0 \mathrm{~V}, 10 \mathrm{mV}, 100 \mathrm{mV}$ and $500 \mathrm{mV}$ at $0.372 \mathrm{~A} \mathrm{~g}^{-1}$, effectively $1 C$ assuming $1 \mathrm{e}^{-}$per $6 \mathrm{C}$, the maximum faradaic capacity of graphite, in $1 \mathrm{M} \mathrm{LiPF}_{6}$ in EC/DEC electrolyte. As expected, the first discharge capacity is anomalously high due to faradaic reduction of the electrolyte to form the SEI at $0.8 \mathrm{~V}$ (Figure 2a). ${ }^{30,31}$ The discharge profiles essentially trace each other until the cutoff voltage is reached, however, a deviation is observed for the $100 \mathrm{mV}$ cutoff cell. This deviation is due to slight inconsistencies between cells possibly caused by differences in the way the loose powders were pressed, for example. Therefore, all subsequent data is shown with error bars that represent the standard deviation between at least three replicate cells. The curves shown in Figure 2 are representative curves.

As long as the discharge cutoff voltage is below the potential at which SEI is formed, which will vary depending on rate and electrolyte composition (vide infra), contributions to the first discharge capacity will be anomalously high due to SEI formation. Most of the charge passed due to SEI formation is irreversible, as evidenced by the much lower charge capacity (Figure 2a). The discharge profile of the subsequent cycle, shown in Figure $2 b$, contains no obvious faradaic plateau and is dominated by a sloping profile. The capacity observed on the second cycle is still somewhat irreversible, although it is more reversible than SEI formation. The discharge and charge profiles of 

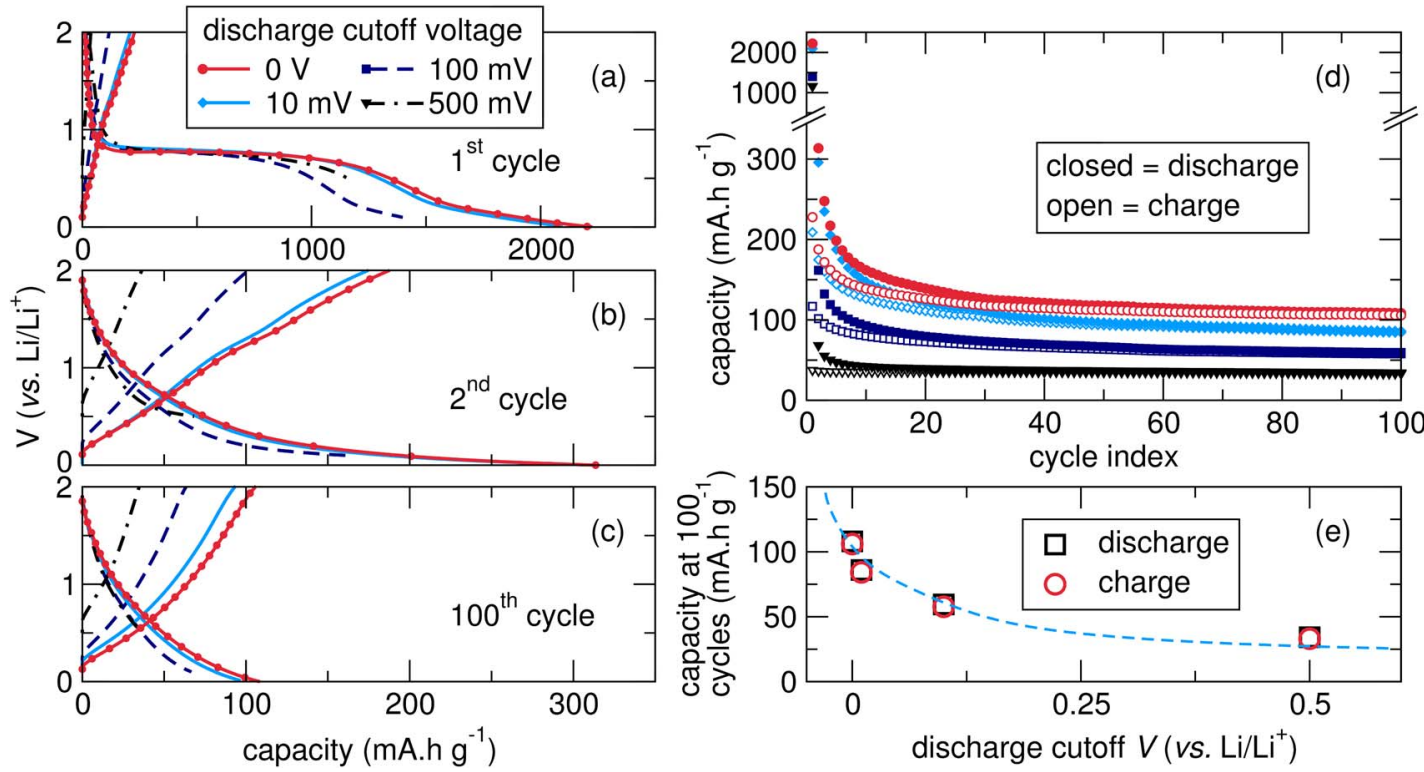

Figure 2. Ketjenblack electrodes cycled at $0.372 \mathrm{~A} \mathrm{~g}^{-1}$ in $1 \mathrm{M} \mathrm{LiPF}_{6}$ in EC/DEC electrolyte with varied discharge cutoff voltages. The charge cutoff is $2 \mathrm{~V}$ in all cases. (a) The first discharge is dominated by the decomposition of the carbonate electrolyte to form the SEI as evidenced by the noticeable faradaic plateau. (b) The $2^{\text {nd }}$ cycle exhibits a sloping discharge profile with a slight tail at lower voltages. (c) The features present in the initial cycles are absent in the $100^{\text {th }}$ cycle. Note that (a) is shown on a different $x$-axis scale compared to (b) and (c). (d) All cells cycle with high initial capacities that fade quickly to a lower but significant reversible capacity. (e) As the cutoff voltage is increased, the discharge and charge capacities exhibit a non-linear trend that matches the shape of the discharge profile. A discharge profile is overlaid to show the relationship between the capacity and the discharge profile.

the $100^{\text {th }}$ cycle are shown in Figure $2 \mathrm{c}$ and display mostly sloping behavior with no apparent inflections or plateaus. The Coulombic efficiency of the $100^{\text {th }}$ cycle is significantly higher than the initial cycles suggesting more reversible charge storage.

At all cutoff voltages, substantial, reversible capacity after 100 cycles is observed after an initial capacity fade during the first 10 cycles (Figure 2d). The initial capacity fade is likely due to some quasi-reversible faradaic activity on the carbon surface due to electrolyte decomposition. Quasi-reversible SEI formation has been suggested previously. ${ }^{21-23}$ Hints of faradaic activity are observed in the $2^{\text {nd }}$ charge and discharge profiles as evidenced by the inflection in the charge profile at around $1.5 \mathrm{~V}$ and the long tail in the discharge profile below $0.5 \mathrm{~V}$ (Figure $2 \mathrm{~b}$ ). A purely capacitive mechanism or a mechanism involving a large distribution of redox active species/sites for Li ions would be void of such features and would show smooth discharge and charge profiles with a consistent slope throughout. Additionally, if no oxidation of the SEI was occurring, then the first charge capacity should more closely match that of the steady state capacity values obtained at higher cycle indexes. Instead, both the discharge and charge capacity gradually fade as the carbons are cycled. This initial equilibration of the SEI layer occurs over the first 20 cycles followed by gradual sloping discharge and charge profiles thereafter. The cells with higher cutoff potentials reach the reversible, steady capacity in fewer cycles.

The magnitude of the reversible, steady capacity is strongly related to the cutoff voltage with higher cutoff voltages leading to lower reversible capacity. The trend between the capacity at 100 cycles and the cutoff voltage is non-linear and closely mimics the shape of the discharge profile, which is overlaid in Figure 2e. This suggests that increasing the cutoff voltage simply prevents access to the charge storage that can occur at the lower potentials.

In addition to evaluating the reversible capacity as a function of cutoff voltage, the effect of cycling rate was also explored. The discharge capacity of Super P and Ketjenblack cycled from $10 \mathrm{mV}$ to $2 \mathrm{~V}$ at $3.72 \mathrm{~A} \mathrm{~g}^{-1}, 0.744 \mathrm{~A} \mathrm{~g}^{-1}, 0.372 \mathrm{~A} \mathrm{~g}^{-1}$, and $0.0372 \mathrm{~A} \mathrm{~g}^{-1}$ in EC/EMC are shown in Figure 3. Ketjenblack supports the highest reversible capacity when cycled at $0.0372 \mathrm{~A} \mathrm{~g}^{-1}$ and exhibits a rate dependence that would be expected from kinetically limited processes. In the Super $\mathrm{P}$ case, the capacity at 100 cycles is less dependent on rate. At $3.72 \mathrm{~A} \mathrm{~g}^{-1}$, both materials show negligible reversible capacity likely due to kinetic limitations at this fast rate. Additionally, the capacity due to SEI formation (cycle 1) is lower than would be expected from a linear trend extrapolated from the first three rates suggesting that the SEI formation is also limited by kinetics at

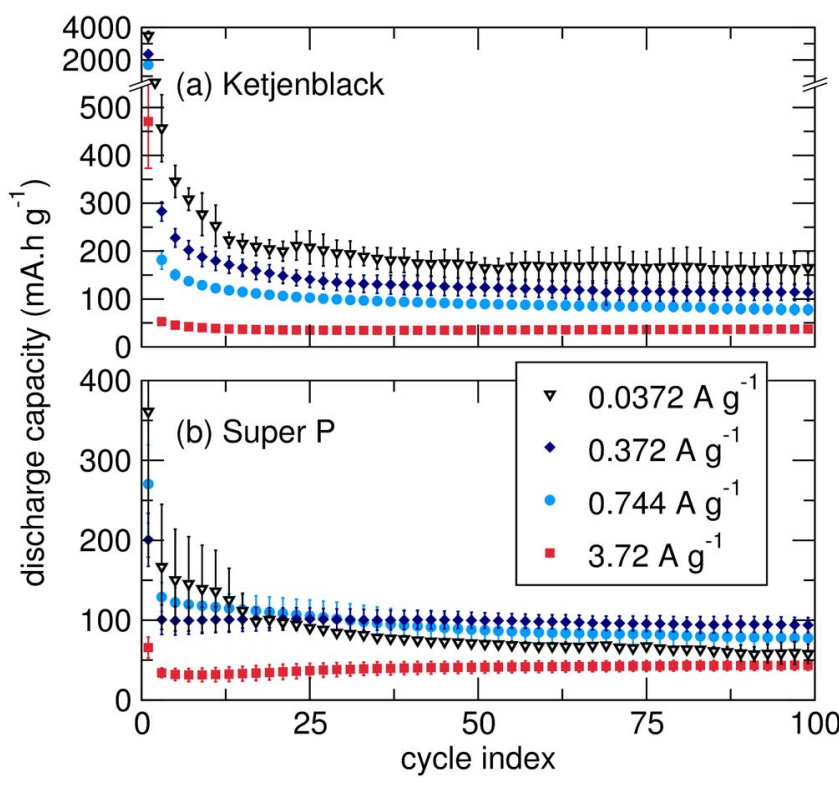

Figure 3. Discharge capacity of (a) Ketjenblack and (b) Super P electrodes cycled at varying rates in $1 \mathrm{M} \mathrm{LiPF}_{6}$ in EC/EMC from $10 \mathrm{mV}$ to $2 \mathrm{~V}$. An average of at least three replicate cells is shown with the standard deviation represented by the error bars. The carbons reach a steady capacity after fewer cycle numbers when cycled at higher rates. 


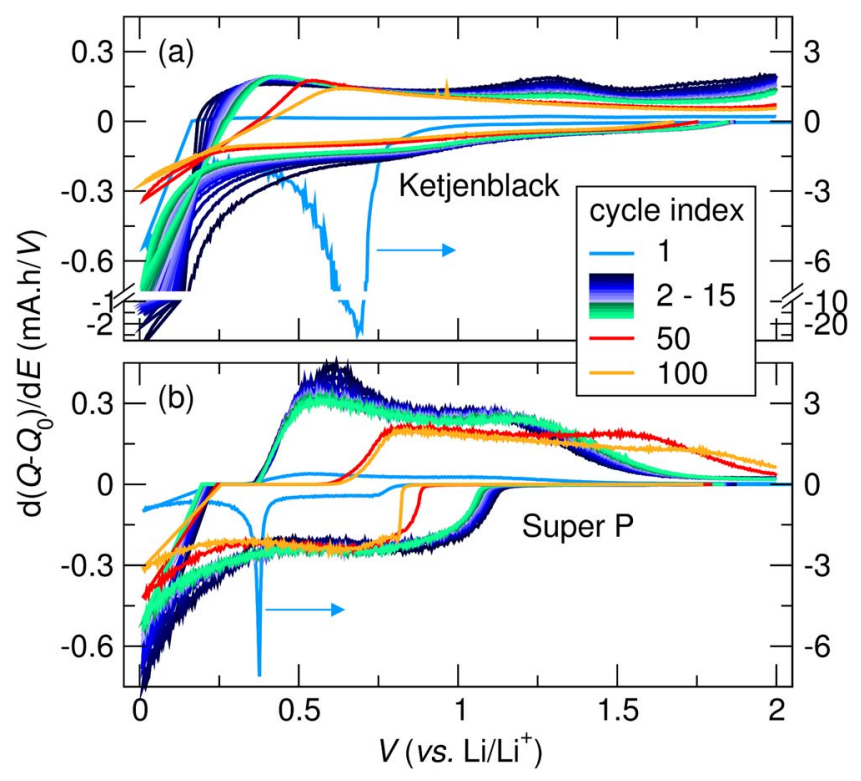

Figure 4. Differential capacity curves of (a) Ketjenblack and (b) Super P cycled between $10 \mathrm{mV}$ and $2 \mathrm{~V}$ at $0.744 \mathrm{~A} \mathrm{~g}^{-1}$ in $1 \mathrm{M} \mathrm{LiPF}_{6}$ in EC/EMC. The first cycle for each both (a) and (b) is plotted on the secondary y-axis (the secondary $y$-axis is $\times 10$ the primary $y$-axis).

$3.27 \mathrm{~A} \mathrm{~g}^{-1}$. Interestingly, the cells cycled at $0.0372 \mathrm{~A} \mathrm{~g}^{-1}$ exhibit lower reversible capacities at higher cycles despite supporting larger initial capacities. The capacity required to form SEI on Ketjenblack is much greater compared to Super $\mathrm{P}$ due to the higher specific surface area of Ketjenblack. Additionally, the gradual capacity fade observed after SEI formation occurs more slowly at lower cycling rates. Therefore, composite electrodes cycled with carbon additives will suffer from higher capacity contributions from the carbon during initial cycles, especially below 20 cycles, when cycled at slow rates. It is also important to note that the capacity above 20 cycles is very stable.

The discharge and charge profiles for each carbon are shown in Figure 4 as differential capacity curves. The first cycle exhibits a sharp peak corresponding to electrolyte reduction to form the SEI. The first cycle is plotted on the secondary y-axis for clarity. The SEI is formed at a more positive potential on Ketjenblack $(0.67 \mathrm{~V})$ compared to Super $\mathrm{P}(0.37 \mathrm{~V})$ suggesting that electrolyte reduction is more facile on Ketjenblack, likely due to the higher surface area. The voltage at which SEI formation occurs is somewhat low for both carbons due to the relatively fast cycling rate of $0.744 \mathrm{~A} \mathrm{~g}^{-1}$. Oxidation is observed on the first charge for both Ketjenblack and Super P, although the broad peak is difficult to observe in Figure 4 due to scaling issues. The first 15 cycles exhibit gradual changes in the discharge and charge profiles which cause the gradual drop in capacity discussed above. The most significant change in the profiles from cycle 2 to cycle 15 is the decrease in capacity contribution at the lowest potentials. Concurrently, the oxidation peak occurring around $1.2 \mathrm{~V}$ decreases steadily. The materials stabilize to a steady, reproducible profile after 50 cycles.

The shapes of the discharge and charge profiles are qualitatively different between the Super P and Ketjenblack. The discharge profile for Super P has a low voltage tail, similar to the Ketjenblack, however, the contribution of the capacitive features are relatively larger. An overpotential associated with the onset of the capacitive storage is observed only for Super P and it increases gradually as the cell cycles on both discharge and charge. The increase in overpotential is the primary cause for capacity fade as Super $\mathrm{P}$ cycles while in the Ketjenblack case, most of the initial capacity fade is due to a decrease in capacity contribution from the low voltage region. Interestingly,

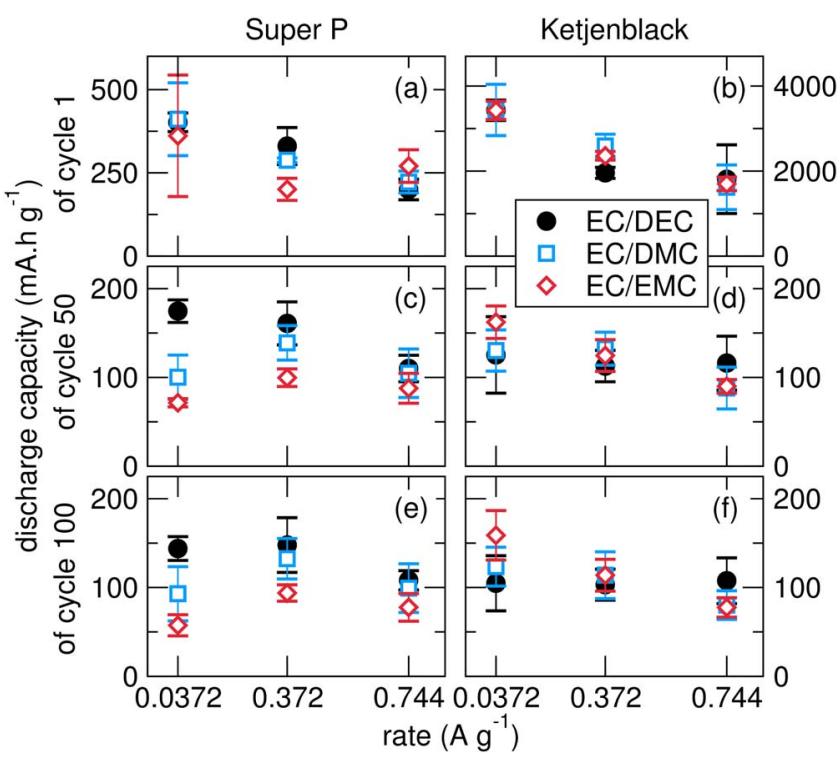

Figure 5. Discharge capacity of Super P (left panels) and Ketjenblack (right panels) electrodes cycled between $10 \mathrm{mV}$ and $2 \mathrm{~V}$ at $0.0372 \mathrm{~A} \mathrm{~g}^{-1}$, $0.372 \mathrm{~A} \mathrm{~g}^{-1}, 0.744 \mathrm{~A} \mathrm{~g}^{-1}$ corresponding to effective $C$ rates of $C / 10, C$, and $2 C$, respectively, assuming $1 \mathrm{e}^{-}$per $6 \mathrm{C}$. The electrolyte is $1 \mathrm{M} \mathrm{LiPF}_{6}$ in the indicated carbonate solvents. The $(\mathrm{a}, \mathrm{b}) 1^{\text {st }},(\mathrm{c}, \mathrm{d}) 50^{\mathrm{th}}$, and $(\mathrm{e}, \mathrm{f}) 100^{\text {th }}$ cycles are shown. Each data point represents the average of duplicate cells with the standard deviation indicated as the error bar.

both Super P and Ketjenblack exhibit two oxidative features during charging.

In order to provide information about the activity of the conductive carbons in electrode slurries for a variety of systems, the carbons were cycled in several EC electrolytes including EC/EMC, EC/DMC, and $\mathrm{EC} / \mathrm{DEC}$ at ratios commonly used in the literature (Figure 5). The first discharge capacity for both Super P and Ketjenblack shows a strong rate dependence in all electrolytes (Figure 5a) due to the faradaic nature of SEI formation. The capacity of the first cycle is significantly higher for Ketjenblack compared to Super P in all electrolytes due to the much higher surface area. The capacity at 50 cycles and 100 cycles is also shown in Figure 5 to convey the reversibility of the charge storage capabilities of the carbon materials after many cycles (Figure $5)$. The reversible capacity observed on Ketjenblack electrodes does not depend on the electrolyte composition. EC/DEC, EC/DMC, and EC/EMC all result in similar capacities from the $50^{\text {th }}$ to $100^{\text {th }}$ cycles. The reversible capacity on Super P electrodes, however, shows a slight dependence on electrolyte composition with the EC/DEC electrolyte resulting in slightly higher capacities followed by EC/DMC and finally EC/EMC. Interestingly, however, the capacity observed at higher cycle numbers is very similar between Ketjenblack and Super P despite their drastically different surface areas. Although the two carbons have different surface areas, the particle size of the two carbons are very similar with the higher surface area of Ketjenblack resulting from the internal surface area provided by hollow particles. The similar capacities at 50 and 100 cycles could therefore be explained by SEI filling up the internal pores of the Ketjenblack leaving only the surface of the particle available for subsequent charge storage.

In all cases, the capacity fade from the $50^{\text {th }}$ to $100^{\text {th }}$ cycle is negligible suggesting that the capacity of the carbons at 50 cycles will persist throughout the cycling life, at least to 100 cycles. The capacity of the carbons at each rate and in all three electrolytes is tabulated in Table I for reference. The minimum reversible capacity generated from the carbons can be considered to be the capacity at the $100^{\text {th }}$ cycle, however, composite electrodes cycled at these low potentials will suffer from larger capacity contributions from the carbons during earlier cycles, as mentioned previously. The capacity is also given in $\mathrm{F} \mathrm{g}^{-1}$ for the $100^{\text {th }}$ cycle to facilitate comparison 
Table I. Discharge capacities in $\mathrm{mAh}^{-1}$ of Super $\mathrm{P}$ and Ketjenblack electrodes cycled between $10 \mathrm{mV}$ and $2 \mathrm{~V}$ in various carbonate electrolytes at varying rates. The effective $C$ rate is given assuming $1 \mathrm{e}^{-}$per $6 \mathrm{C}$. The error represents the standard deviation of at least three replicate cells.

Super P

discharge capacity $\left(\mathrm{mAh} \mathrm{g}^{-1}\right)$

\begin{tabular}{|c|c|c|c|c|c|c|c|}
\hline \multirow{2}{*}{ rate $\left(\mathrm{A} \mathrm{g}^{-1}\right)$} & \multirow[b]{2}{*}{ eff. $C$ rate } & & & & \\
\hline & & $1^{s t}$ cycle & $50^{\text {th }}$ cycle & $100^{\text {th }}$ cycle & $1^{s t}$ cycle & $50^{\text {th }}$ cycle & $100^{\text {th }}$ cycle \\
\hline \multicolumn{8}{|c|}{ ethylene carbonate/diethyl carbonate (EC/DEC) } \\
\hline 0.0372 & $C / 10$ & $402 \pm 28$ & $175 \pm 13$ & $144 \pm 13$ & $3430 \pm 240$ & $125 \pm 43$ & $105 \pm 31$ \\
\hline 0.372 & $C$ & $331 \pm 56$ & $161 \pm 24$ & $148 \pm 31$ & $1960 \pm 130$ & $113 \pm 18$ & $103 \pm 18$ \\
\hline \multicolumn{8}{|c|}{ ethylene carbonate/dimethyl carbonate (EC/DMC) } \\
\hline 0.0372 & $C / 10$ & $411 \pm 109$ & $100 . \pm 25$ & $93.0 \pm 30.5$ & $3440 \pm 600$ & $130 \pm 23$ & $124 \pm 22$ \\
\hline 0.372 & $C$ & $288 \pm 7$ & $139 \pm 19$ & $132 \pm 23$ & $2590 \pm 280$ & $132 \pm 19$ & $114 \pm 26$ \\
\hline \multicolumn{8}{|c|}{ ethylene carbonate/ethyl methyl carbonate (EC/EMC) } \\
\hline 0.0372 & $C / 10$ & $361 \pm 182$ & $71.5 \pm 4.6$ & $57.5 \pm 11.9$ & $3430 \pm 220$ & $162 \pm 18$ & $159 \pm 28$ \\
\hline 0.372 & $C$ & $200 . \pm 33$ & $99.7 \pm 9.9$ & $93.8 \pm 9.3$ & $2360 \pm 103$ & $125 \pm 18$ & $114 \pm 18$ \\
\hline 0.744 & $2 C$ & $270 \pm 49$ & $87.8 \pm 16.8$ & $77.7 \pm 15.6$ & $1700 \pm 160$ & $89.9 \pm 7.6$ & $77.6 \pm 11.0$ \\
\hline 3.72 & $10 C$ & $65.6 \pm 13.2$ & $41.0 \pm 7.6$ & $43.5 \pm 6.1$ & $471 \pm 98$ & $35.2 \pm 3.3$ & $37.3 \pm 4$ \\
\hline
\end{tabular}

with materials intended for capacitor or supercapacitor applications (Table II).

Comparing the electrochemical impedance spectroscopy (EIS) of the two carbon additives in all three electrolyte compositions before and after cycling gives further insight into the performance of the materials and the nature of the SEI. The EIS data is represented as Nyquist plots with the real part of the impedance $\left(Z^{\prime}\right)$ plotted on the $x$-axis vs. the negative imaginary part of the impedance $\left(-Z^{\prime \prime}\right)$ on the y-axis in Figure 6. Nyquist plots can be fit with equivalent circuit models in order to obtain resistance values corresponding to physical processes, however, here we will only consider a qualitative comparison of the Nyquist plots. The semicircle at low $Z^{\prime}$ (high frequency) can be represented by a capacitor and a resistor in parallel modeling the interface between the carbon and the electrolyte. The radius of the semicircle is related to the charge trans-

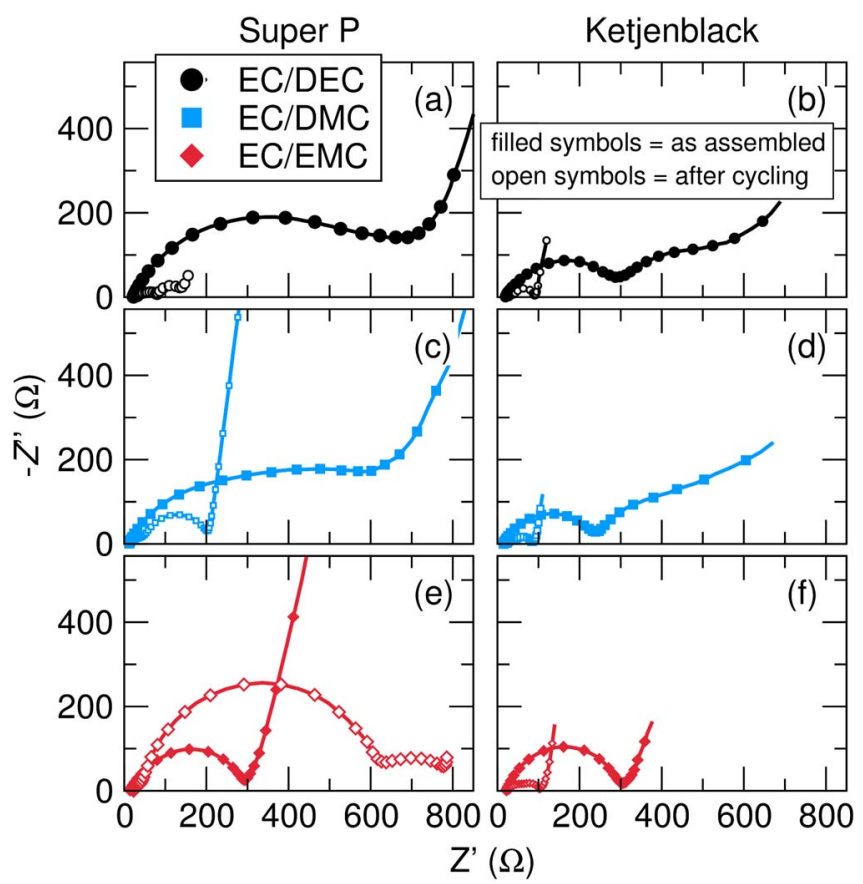

Figure 6. Nyquist plots before and after cycling of Super P (left panels) and Ketjenblack (right panels) in all three electrolyte compositions. The cells were cycled for 100 cycles at $0.744 \mathrm{~A} \mathrm{~g}^{-1}$ between $10 \mathrm{mV}$ and $2 \mathrm{~V}$. fer resistance of the electrode. ${ }^{32}$ Before cycling, the charge transfer resistance of the Ketjenblack electrodes is much less than that of the Super P electrodes in all electrolytes suggesting that the electrolyte is able to wet the Ketjenblack more efficiently (Figure 6). The enhanced wettability is likely due to the higher surface area of the Ketjenblack which increases the carbon-electrolyte interfacial area.

Due to the strong influence of surface area on the Nyquist plots, it is most informative to compare the change in the EIS after cycling when evaluating differences between the two carbons. In the Ketjenblack case, the cycled cell exhibits a much lower impedance compared to the uncycled cell. The Super P cells also exhibit a decrease in impedance but not as significant as that of Ketjenblack. The only condition that results in higher impedance after cycling is Super $\mathrm{P}$ cycled in the EC/EMC electrolyte. Although only one cell is shown in Figure 6c, this inverse relationship was observed for all Super P electrodes cycled in EC/EMC. Cycling Super P in EC/EMC also results in lower capacities compared to the other two electrolyte compositions (Figure 5). Additionally, Super P cells cycled in EC/DEC exhibit lower charge transfer resistances after cycling which could be the cause for the higher reversible capacity observed in this electrolyte.

In order to investigate the effect of the composition of the SEI on the reversible capacity in more detail, the electrolyte composition was modulated by changing the relative concentration of EC to DMC. When Super $\mathrm{P}$ is cycled in electrolytes of varied composition at 0.372 $\mathrm{A} \mathrm{g}^{-1}$, reversible capacity is observed even in $100 \%$ DMC electrolyte (Figure 7). The SEI plateau on the first cycle is absent for the $100 \%$ DMC case confirming that the faradaic plateau is mostly due to EC reduction (Figure 7a). Although the plateau is absent, the 100\% DMC electrolyte still exhibits a high initial discharge capacity indicating that SEI formation is occurring despite the absence of EC. Interestingly, the $100 \%$ DMC electrolyte also exhibits the lowest reversible capacity (Figure 7c). This could be due to the change in composition of the SEI in the absence of EC. For example, the SEI in the 100\% DMC electrolyte could be more resistive, however, higher resistances should increase the hysteresis between the discharge and charge curves, which is not observed. The cells that show the largest hysteresis, which can be attributed to SEI resistance, during the $25^{\text {th }}$ cycle are the cells cycled in the $88 \%$ DMC electrolyte composition. The $50 \%$ and $70 \%$ DMC compositions result in the thickest SEI as evidenced by the high gravimetric capacity for the $1^{\text {st }}$ cycle. These cells are also the cells which have the highest reversible capacity thereafter suggesting that SEI formation is indeed important for the charge storage of Super $\mathrm{P}$. In order to prevent this process, it is necessary to cycle composite electrodes to cutoff voltages above the voltage at which SEI formation occurs. 

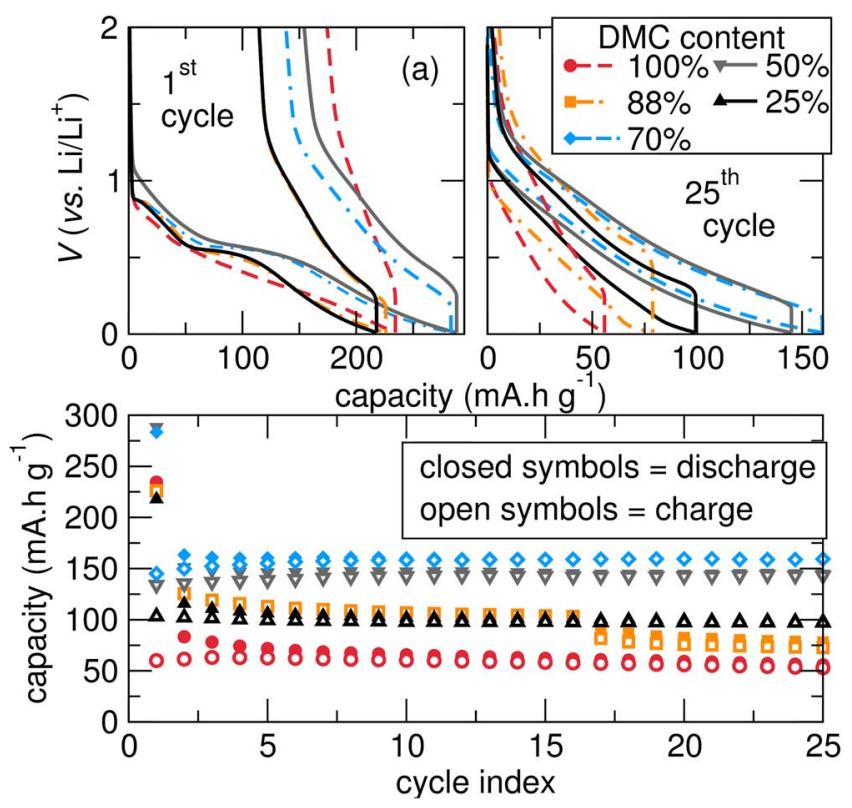

Figure 7. Super $\mathrm{P}$ cycled in DMC at $0.372 \mathrm{~A} \mathrm{~g}^{-1}$ with varying EC content, as indicated. (a) The first discharge plateau, which corresponds to electrolyte decomposition, is lost when Super P is cycled in 100\% DMC suggesting the plateau is due to EC reduction. (b) Capacitive storage on Super P in higher DMC content electrolytes exhibits worse hysteresis probably due to a more resistive SEI layer formed during the $1^{\text {st }}$ cycle. (c) Super $\mathrm{P}$ is able to cycle reversibly in all electrolytes. 70\% DMC is the optimal electrolyte composition for the reversible capacity.

\section{Conclusions}

Super P and Ketjenblack conductive carbon additives are shown here to exhibit substantial, steady capacity in carbonate-based electrolytes when cycled at low potentials (below $1 \mathrm{~V}$ ). We have tabulated the capacity that can be expected of both carbon additives at several rates in a variety of carbonate electrolyte compositions. Cells cycled at slower rates are found to incur a higher capacity contribution from the carbon additives during the first 20 cycles due to the longer time periods spent at the low voltages where electrolyte decomposition, and SEI formation, occurs. Those cycled at faster rates, $\geq 0.372 \mathrm{~A} \mathrm{~g}^{-1}$,

Table II. Discharge capacities of the $100^{\text {th }}$ cycle in $\mathrm{F} \mathrm{g}^{-1}$ of Super $P$ and Ketjenblack electrodes cycled between $10 \mathrm{mV}$ and $2 \mathrm{~V}$ in carbonate electrolytes at varying rates. The effective $C$ rate is given assuming $1 \mathrm{e}^{-}$per $6 \mathrm{C}$. The error represents the standard deviation of at least three replicate cells.

\begin{tabular}{|c|c|c|c|}
\hline \multirow{2}{*}{$\begin{array}{l}\text { rate } \\
\left(\mathrm{Ag}^{-1}\right)\end{array}$} & \multirow{2}{*}{$\begin{array}{l}\text { eff. } \\
C \text { rate }\end{array}$} & \multicolumn{2}{|c|}{$\begin{array}{l}\text { discharge capacity of } \\
100^{\text {th }} \text { cycle }\left(\mathrm{F} \mathrm{g}^{-1}\right)\end{array}$} \\
\hline & & Super $P$ & Ketjentblack \\
\hline \multicolumn{4}{|c|}{ ethylene carbonate/diethyl carbonate (EC/DEC) } \\
\hline 0.0372 & $C / 10$ & $261 \pm 24$ & $190 . \pm 56$ \\
\hline 0.372 & $C$ & $268 \pm 56$ & $186 \pm 32$ \\
\hline 0.744 & $2 C$ & $195 \pm 20$ & $195 \pm 47$ \\
\hline \multicolumn{4}{|c|}{ ethylene carbonate/dimethyl carbonate (EC/DMC) } \\
\hline 0.0372 & $C / 10$ & $168 \pm 55$ & $224 \pm 40$ \\
\hline 0.372 & $C$ & $239 \pm 42$ & $206 \pm 47$ \\
\hline 0.744 & $2 C$ & $180 \pm 50$ & $145 \pm 29$ \\
\hline \multicolumn{4}{|c|}{ ethylene carbonate/ethyl methyl carbonate (EC/EMC) } \\
\hline 0.0372 & $C / 10$ & $104 \pm 22$ & $288 \pm 51$ \\
\hline 0.372 & $C$ & $170 . \pm 17$ & $206 \pm 33$ \\
\hline 0.744 & $2 C$ & $141 \pm 28$ & $140 . \pm 20$ \\
\hline 3.72 & $10 C$ & $78.7 \pm 11$ & $67.5 \pm 7.7$ \\
\hline
\end{tabular}

exhibit high capacity due to SEI formation on the carbons on the first cycle followed by a stable but nevertheless substantial reversible capacity. The formation of the SEI appears to be important in stabilizing the capacity of the carbons over many cycles. In order to prevent the activity of the carbons, composite electrodes must be cycled above the potential at which SEI formation occurs (about $1 \mathrm{~V}$ ) as SEI formation stabilizes subsequent reversible capacity. This potential could vary depending on the composition of the composite electrode, the electrolyte composition, and cycling rate. Cycling electrodes above the potential at which SEI formation occurs may not be realistic, however, due to the low operating voltage of viable alternative anodes. In this case, Table I serves as a reference and allows for a rough deconvolution of the observed capacity in a composite electrode. These values are especially important to consider for alternative anode materials that are insulating, and therefore require a higher percentage of carbon in the composite electrode.

\section{Acknowledgments}

K. A. S. acknowledges Fred Wudl for helpful discussions. K. A. S. acknowledges graduate fellowship support from the ConvEne IGERT Program of the National Science Foundation (DGE 0801627) and postdoctoral funding from the St. Elmo Brady Future Faculty Postdoctoral Fellowship. The experiments were performed in Materials Research Laboratory facilities at UCSB, supported by the MRSEC Program of the NSF under grant No. DMR 1121053. M. A. L. thanks the RISE program (NSF DMR 1121053) for support. C. P. G. thanks the Assistant Secretary for Energy Efficiency and Renewable Energy, Office of Vehicle Technologies of the U. S. Department of Energy under Contract No. DE-AC02-05CH11231, under the Batteries for Advanced Transportation Technologies (BATT) Program subcontract \#7057154 for support.

\section{References}

1. R. A. Sharma and R. N. Seefurth, J. Electrochem. Soc., 123, 1763 (1976)

2. B. A. Boukamp, G. C. Lesh, and R. A. Huggins, J. Electrochem. Soc., 128, 725 (1981).

3. M. V. Reddy, G. V. Subba Rao, and B. V. R. Chowdari, Chem. Rev., 113, 5364 (2013).

4. P. Poizot, S. Laruelle, S. Grugeon, L. DuPont, and J.-M. Tarascon, Nature, 407, 496 (2000).

5. M. M. Butala, K. R. Danks, M. A. Lumley, S. Zhou, B. C. Melot, and R. Seshadri, ACS Appl. Mater. Interfaces, 8, 6496 (2016).

6. F. Wang, R. Robert, N. A. Chernova, N. Pereira, F. Omenya, F. Badway, X. Hua, M. Ruotolo, R. Zhang, L. Wu, V. Volkov, D. Su, B. Key, M. S. Whittingham, C. P. Grey, G. G. Amatucci, Y. Zhu, and J. Graetz, J. Am. Chem. Soc., 133, 18828 (2011).

7. F. Badway, A. N. Mansour, N. Pereira, J. F. Al-Sharab, F. Cosandey, I. Plitz, and G. G. Amatucci, Chem. Mater, 19, 4129 (2007).

8. Y. Oumellal, A. Rougier, G. A. Nazri, J.-M. Tarascon, and L. Aymard, Nat. Mater. 7, 916 (2008).

9. S. Britto, M. Leskes, X. Hua, C.-A. Hbert, H. S. Shin, S. Clarke, O. Borkiewicz, K. W. Chapman, R. Seshadri, J. Cho, and C. P. Grey, J. Am. Chem. Soc., 137, 8499 (2015).

10. A. Debart, L. Dupont, R. Patrice, and J. M. Tarascon, Solid State Sci., 8, 640 (2006).

11. J. K. Hong, J. H. Lee, and S. M. Oh, J. Power Sources, 111, 90 (2002).

12. J. Jamnik and J. Maier, Phys. Chem. Chem. Phys., 5, 5215 (2003).

13. P. Balaya, H. Li, L. Kienle, and J. Maier, Adv. Funct. Mater., 13, 621 (2003).

14. L. Fransson, T. Eriksson, K. Edstrm, T. Gustafsson, and J. O. Thomas, J. Power Sources, 101, 1 (2001).

15. S. Flandrois and B. Simon, Carbon, 37, 165 (1999).

16. M. Yoshio, H. Wang, K. Fukuda, Y. Hara, and Y. Adachi, J. Electrochem. Soc., 147, 1245 (2000)

17. A. G. Pandolfo and A. F. Hollenkamp, J. Power Sources, 157, 11 (2006).

18. F. Beck, M. Dolata, E. Grivei, and N. Probst, J. Appl. Electrochem., 31, 845 (2001).

19. D. A. Stevens and J. R. Dahn, J. Electrochem. Soc., 148, A803 (2001).

20. O. Y. Chusid, E. Ein Ely, D. Aurbach, M. Babai, and Y. Carmeli, J. Power Sources, 43, 47 (1993).

21. R. Yazami and P. A. Touzain, J. Power Sources, 9, 365 (1983).

22. J. R. Dahn, T. Zheng, Y. Liu, and J. S. Xue, Science, 270, 590 (1995).

23. A. L. Michan, M. Leskes, and C. P. Grey, Chem. Mater, 28, 385 (2015)

24. T. Xing, T. Ramireddy, L. H. Li, D. Gunzelmann, H. Zeng, W. Qi, S. Zhou, and Y. Chen, Phys. Chem. Chem. Phys., 17, 5084 (2015).

25. J. Syzdek, M. Marcinek, and R. Kostecki, J. Power Sources, 245, 739 (2014). 
26. R. Younesi, A. S. Christiansen, R. Scipioni, D.-T. Ngo, S. B. Simonsen, K. Edstrm, J. Hjelm, and P. Norby, J. Electrochem. Soc., 162, A1289 (2015).

27. X.-H. Yang, P. He, and Y.-Y. Xia, Electrochem. Comm., 11, 1127 (2009).

28. W. F. Verhelst, K. G. Wolthuis, A. Voet, P. Ehrburger, and J. B. Donnet, Rubber Chem. Technol., 50, 735 (1977).

29. E. Peled, J. Electrochem. Soc., 126, 2047 (1979).
30. D. Aurbach, B. Markovsky, I. Weissman, E. Levi, and Y. Ein-Eli, Electrochim. Acta, 45, 67 (1999)

31. P. Novak, F. Joho, R. Imhof, J.-C. Panitz, and O. Haas, J. Power Sources, 81-82, 212 (1999).

32. Y.-J. Choi, Y.-D. Chung, C.-Y. Baek, K.-W. Kim, H.-J. Ahn, and J.-H. Ahn, J. Power Sources, 184, 548 (2008). 